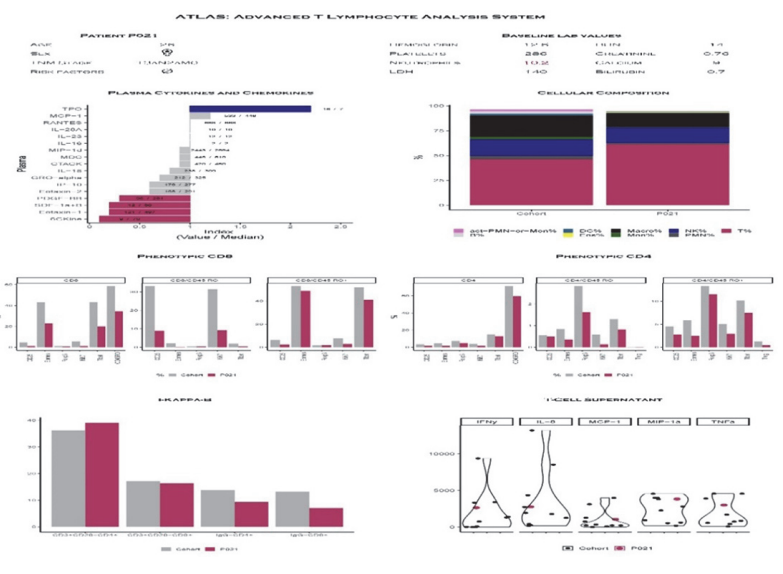

Abstract 52 Figure 2 Patient 021 dashboard

compared patients' percentages to sample average relative abundance. Two patients, P011 shown in figure 1 and P021 shown in figure 2, are depicted using this approach.

Conclusions ATLAS can be used in real-world conditions to generate comprehensive immunological profiles of cancer patients. Individual profiles indicate that immunological constitution is heterogeneous among patients, even with the same tumor type. We propose that the addition of ATLAS to our clinical and immunological toolbox may help stratify patients to articulate truly personalized oncologic therapies.

Ethics Approval The study was approved by Loyola University Medical Center and Loyola University Chicago Ethics Board and Institutional Review Board, approval number 209364.

http://dx.doi.org/10.1136/jitc-2020-SITC2020.0052

\section{PREDICTORS OF RESPONSE TO IMMUNE CHECKPOINT INHIBITOR THERAPY IN METASTATIC SOLID TUMORS: REAL WORLD EVIDENCE}

Aasems Jacob*, Jianrong Wu, Jill Kolesar, Eric Durbin, Aju Mathew, Susanne Arnold, Aman Chauhan. University of Kentucky, Lexington, KY, USA

Background Immune checkpoint inhibitor (ICI) therapy is increasingly being used in oncology and novel predictive biomarker for efficacy and side effects are an unmet need. ${ }^{1} 2$ The study aims to do a comprehensive analysis of factors affecting outcome from ICI therapy with real-world data and identify potential predictive biomarkers in diverse populations. Methods We performed a retrospective analysis of patients with metastatic solid tumors who received ICI and underwent molecular profiling with FoundationOne ${ }^{\circledR}$ CDx panel between 2016 and 2020 at Markey Cancer Center, Lexington KY. Progression-free survival (PFS), radiological response, and autoimmune side effects were analyzed and compared with various molecular biomarkers (figure 1). Logistic regression, Fisher's exact test, Kaplan-Meier method, log-rank test, and Cox regression were used to analyze clinical features and efficacy outcomes.

Results 69 patients were included in the study (tables 1 and 2). A statistically significant improvement in PFS was observed in the PIK3 mutated cohort (median 123 vs. 23 weeks. $\mathrm{HR}=2.51$. 95\% $\mathrm{CI} 1.23,5.14$; table 3 and figure 2). This was independent of tumor mutational burden (TMB) status or PDL1 expression status (HR 3.24, $\mathrm{p}=0.016$ ). PIK3 mutants had a higher overall response rate (ORR) than the wild type
(69.6\% vs. $43.5 \%$, OR $0.34 ; p=0.045$; tables 3 and 4 ). PIK3 mutants had a higher risk of developing immune-related adverse events (IRAEs) $(73.9 \%$ vs. 37\%, p=0.004). PIK3 mutation did not associate with TMB, PDL1 expression or microsatellite stability status. Median PFS was higher in the high TMB cohort compared to the low-intermediate group and reached statistical significance (median not reached vs. 26

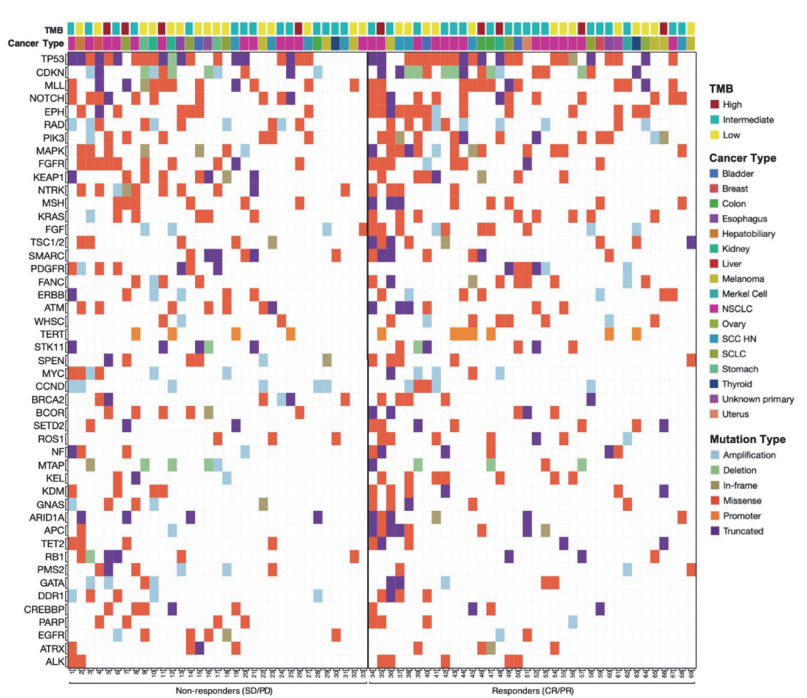

Abstract 53 Figure 1 Mutational analysis of patients receiving immunotherapy grouped based on radiologic response, in the order of mutational load and frequency of mutations

Abstract 53 Table 1 Baseline characteristics of the study population

\begin{tabular}{|ll|}
\hline Characteristics & Total (\%) \\
\hline Age group & \\
$18-64$ & $40(58)$ \\
$65-74$ & $19(27.5)$ \\
$\geq 75$ & $10(14.5)$ \\
\hline Male: Female & $36(52.2): 33(47.8)$ \\
\hline Race & \\
Caucasian & $63(91.3)$ \\
African American & $4(5.8)$ \\
Hispanic & $1(1.4)$ \\
Asian & $1(1.4)$ \\
\hline Cancer Site & \\
NSCLC & $26(37.7)$ \\
SCC Head \& Neck & $8(11.6)$ \\
Melanoma & $7(10.1)$ \\
Gastrointestinal & $7(10.1)$ \\
Kidney/Bladder & $6(8.6)$ \\
Small Cell lung Cancer & $5(7.2)$ \\
Hepatobiliary & $2(2.9)$ \\
Breast & $2(2.9)$ \\
Ovary and uterus & $2(2.9)$ \\
Thyroid & $2(2.9)$ \\
Merkel Cell & $1(1.4)$ \\
Unknown primary & $1(1.4)$ \\
\hline Tobacco use & \\
Current/previous use & $54(78.3)$ \\
Never used & $15(21.7)$ \\
\hline NSCLC: Non-small cell lung cancer, SCC: Squamous \\
cell carcinoma & \\
\hline
\end{tabular}


Abstract 53 Table 2 Treatment and biomarker characteristics of study population

\begin{tabular}{|l|l|}
\hline Characteristics & $\mathbf{n}(\%)$ \\
\hline Immunotherapy treatment & \\
Chemoimmunotherapy & $6(8.7)$ \\
Combination immunotherapy & $10(14.5)$ \\
Single agent immunotherapy & $53(76.8)$ \\
\hline ICl used & \\
Atezolizumab & $2(2.9)$ \\
Ipilimumab-Nivolumab & $11(15.9)$ \\
Nivolumab & $11(15.9)$ \\
Pembrolizumab & $45(65.2)$ \\
\hline IRAEs & \\
Any & $33(47.8)$ \\
Grade 1 & $7(10.1)$ \\
Grade 2 & $16(23.2)$ \\
Grade 3/4 & $10(14.4)$ \\
\hline Tumor Mutational Burden & \\
High & $9(13)$ \\
Intermediate & $32(46.4)$ \\
Low & $28(40.6)$ \\
\hline PDL1 tumor proportion score & \\
0 & $14(20.3)$ \\
1-49\% & $19(27.5)$ \\
250 & $10(14.5)$ \\
Unknown & $26(37.7)$ \\
\hline Microsatellite stability & \\
Stable & $62(89.9)$ \\
Unstable & $5(7.2)$ \\
Unknown & $2(2.9)$ \\
\hline Best Response & $14(20.3)$ \\
CR & $22(31.9)$ \\
PR & $12(17.4)$ \\
SD & $21(30.4)$ \\
PD & \\
\hline
\end{tabular}
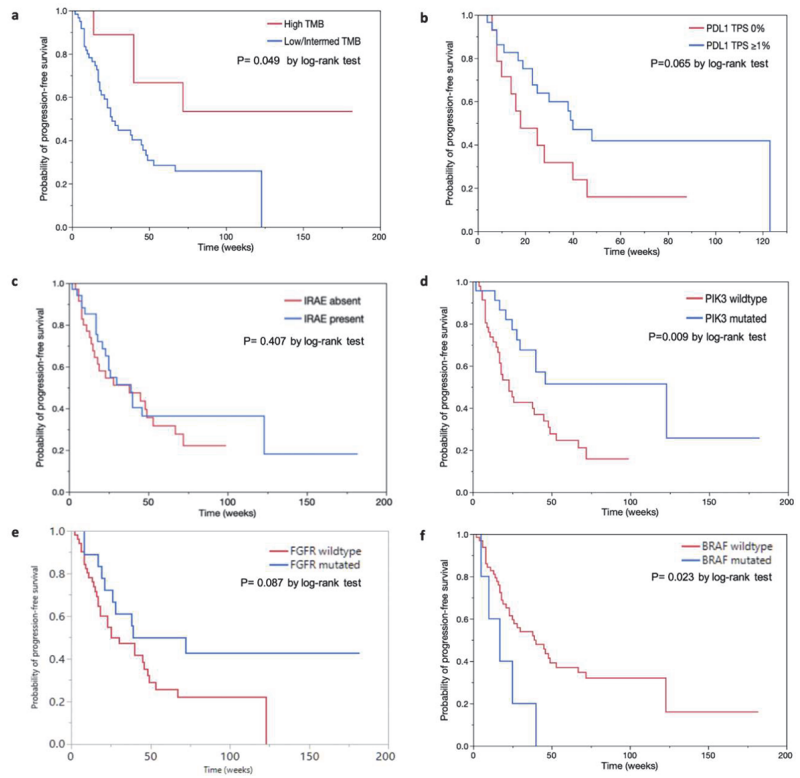

Abstract 53 Figure 2 Kaplan-Meier graphs depicting progression free survival in patients based on tumor samples showing (a) High TMB and low/intermediate TMB; (b) PDL1 expression; (c) Presence of IRAEs; (d) Presence of PIK3 mutation; (e) Presence of FGFR mutation; ( $f$ ) Presence of BRAF mutation
Abstract 53 Table 3 ORR based on various factors with odds ratio calculated using logistic regression model

\begin{tabular}{|c|c|c|c|c|c|}
\hline Variable & Total & $\begin{array}{l}\text { Responders } \\
\text { (CR/PR) n (\%) }\end{array}$ & $\begin{array}{l}\text { Non-responders } \\
\text { (SD/PD) n (\%) }\end{array}$ & $\begin{array}{l}\text { Overall } \\
\text { response rate }\end{array}$ & OR 95\% Cl \\
\hline $\begin{array}{l}\text { All patients } \\
\text { Age }<65\end{array}$ & & $\begin{array}{l}36(52.2) \\
19(47.5)\end{array}$ & $\begin{array}{l}33(47.8) \\
21(52.5)\end{array}$ & $\begin{array}{l}52.2 \% \\
47.5 \%\end{array}$ & \\
\hline $\begin{array}{l}\text { Age }<<5 \\
\text { Age }>65\end{array}$ & $\begin{array}{l}40 \\
29\end{array}$ & $\begin{array}{l}19(47.5) \\
17(58.6)\end{array}$ & $\begin{array}{l}21(52.5) \\
12(41.4)\end{array}$ & $\begin{array}{l}47.5 \% \\
58.6 \%\end{array}$ & $0.64(0.24-1.68)$ \\
\hline $\begin{array}{l}\text { Male } \\
\text { Female }\end{array}$ & $\begin{array}{l}36 \\
33\end{array}$ & $\begin{array}{l}16(44.4) \\
20(60.6)\end{array}$ & $\begin{array}{l}20(55.6) \\
13(39.4)\end{array}$ & $\begin{array}{l}44.4 \% \\
60.6 \%\end{array}$ & $1.92(0.74-5.02)$ \\
\hline $\begin{array}{l}\text { Smoker } \\
\text { Non-smoker }\end{array}$ & $\begin{array}{l}54 \\
15\end{array}$ & $\begin{array}{l}26(48.1) \\
10(66.7)\end{array}$ & $\begin{array}{l}28(51.9) \\
5(33.1)\end{array}$ & $\begin{array}{l}48.1 \% \\
66.7 \%\end{array}$ & $2.15(0.64-7.14)$ \\
\hline $\begin{array}{l}\text { IRAE present } \\
\text { IRAE absent }\end{array}$ & $\begin{array}{l}34 \\
35\end{array}$ & $\begin{array}{l}19(55.9) \\
17(48.6)\end{array}$ & $\begin{array}{l}15(44.1) \\
18(51.4)\end{array}$ & $\begin{array}{l}55.9 \% \\
48.6 \%\end{array}$ & $0.75(0.29-1.92)$ \\
\hline $\begin{array}{l}\text { High TMB } \\
\text { Low/int TMB }\end{array}$ & $\begin{array}{l}9 \\
60\end{array}$ & $\begin{array}{l}5(55.6) \\
31(51.7)\end{array}$ & $\begin{array}{l}4(44.4) \\
29(48.3)\end{array}$ & $\begin{array}{l}55.6 \% \\
51.7 \%\end{array}$ & $1.17(0.29-4.78)$ \\
\hline $\begin{array}{l}\mathrm{TMB}<10 \\
\mathrm{TMB} \geq 10\end{array}$ & $\begin{array}{l}46 \\
23\end{array}$ & $\begin{array}{l}24(52.2) \\
12(52.2)\end{array}$ & $\begin{array}{l}22(47.8) \\
11(47.8)\end{array}$ & $\begin{array}{l}52.2 \% \\
52.2 \%\end{array}$ & $1.00(0.36-2.72)$ \\
\hline $\begin{array}{l}\text { PDL1 0\% } \\
\text { PDL1 >1\% }\end{array}$ & $\begin{array}{l}14 \\
29\end{array}$ & $\begin{array}{l}5(35.7) \\
16(55.2)\end{array}$ & $\begin{array}{l}9(64.3) \\
13(44.8)\end{array}$ & $\begin{array}{l}35.7 \% \\
55.2 \%\end{array}$ & $0.45(0.12-1.68)$ \\
\hline $\begin{array}{l}\text { PIK3 mutated } \\
\text { PIK3 wildtype }\end{array}$ & $\begin{array}{l}23 \\
46\end{array}$ & $\begin{array}{l}16(69.6) \\
20(43.5)\end{array}$ & $\begin{array}{l}7(30.4) \\
26(56.5)\end{array}$ & $\begin{array}{l}69.6 \% \\
43.5 \%\end{array}$ & $0.34(0.12-0.97)$ \\
\hline $\begin{array}{l}\text { FGFR mutated } \\
\text { FGFR wildtype }\end{array}$ & $\begin{array}{l}16 \\
51\end{array}$ & $\begin{array}{l}13(72.2) \\
23(45.1)\end{array}$ & $\begin{array}{l}5(27.8) \\
28(54.9)\end{array}$ & $\begin{array}{l}72.2 \% \\
45.1 \%\end{array}$ & $0.32(0.98-1.01)$ \\
\hline $\begin{array}{l}\text { ROS1 mutated } \\
\text { ROS1 wildtype }\end{array}$ & $\begin{array}{l}10 \\
59\end{array}$ & $\begin{array}{l}9(90.0) \\
27(45.8)\end{array}$ & $\begin{array}{l}1(10.0) \\
32(54.2)\end{array}$ & $\begin{array}{l}90.0 \% \\
45.8 \%\end{array}$ & $0.09(0.01-0.79)$ \\
\hline $\begin{array}{l}\text { BRAF mutated } \\
\text { BRAF wildtype }\end{array}$ & $\begin{array}{l}5 \\
64\end{array}$ & $\begin{array}{l}3(60.0) \\
33(51.6)\end{array}$ & $\begin{array}{l}2(40.0) \\
31(48.4)\end{array}$ & $\begin{array}{l}60.0 \% \\
51.6 \%\end{array}$ & $0.71(0.11-4.54)$ \\
\hline $\begin{array}{l}\text { STK } 11 \text { mutation } \\
\text { STK11 wildtype }\end{array}$ & $\begin{array}{l}11 \\
58\end{array}$ & $\begin{array}{l}4(36.4) \\
32(55.2)\end{array}$ & $\begin{array}{l}7(63.6) \\
26(44.8)\end{array}$ & $\begin{array}{l}36.4 \% \\
55.2 \%\end{array}$ & $2.15(0.57-8.17)$ \\
\hline
\end{tabular}

Abstract 53 Table 4 Identified PIK3 mutations in tumor samples, with their chromosomal position and protein changes

\begin{tabular}{|c|c|c|c|c|c|c|c|}
\hline $\begin{array}{l}\text { Tumor } \\
\text { sample }\end{array}$ & Mutation & Type & Position & Event & $\begin{array}{l}\text { Protein } \\
\text { change }\end{array}$ & TMB & MSI \\
\hline & PIK3CB & Missense & chr3:138426129 & $1402 G>A$ & & high & MSS \\
\hline \multirow[t]{2}{*}{2} & PIK3C2G & CNA Amp & $\begin{array}{l}\text { chr12:18435015- } \\
18800962\end{array}$ & & & intermediate & MSI-L \\
\hline & РІКЗСА & CNA Amp & $\begin{array}{l}\text { chr3:178875270- } \\
178996445\end{array}$ & & & intermediate & MSI-L \\
\hline 3 & PIK3C2G & Missense & chr12:18524203 & $1715 \mathrm{C}>\mathrm{T}$ & A572V & high & MSS \\
\hline 4 & PIK3CA & Missense & chr3:178922364 & $1133 G>T$ & C378F & intermediate & MSS \\
\hline 5 & PIK3C2B & Inframe & chr1:204438234 & 694_696delAAT & N232del & low & MSS \\
\hline 6 & PIK3CB & Truncation & chr3:138452282 & $973-2 A>T$ & $\begin{array}{l}\text { splice site } \\
973-2 A>T\end{array}$ & high & MSS \\
\hline 7 & PIK3CA & Inframe & chr3:178916940 & 328_330delGAA & E110del & intermediate & MSS \\
\hline 8 & PIK3CA & Missense & chr3:178936082 & $1624 \mathrm{G}>\mathrm{A}$ & E542K & low & MSS \\
\hline \multirow[t]{2}{*}{9} & PIK3C2B & Missense & chr1:204438345 & $586 \mathrm{G}>A$ & E196K & intermediate & MSS \\
\hline & PIK3CA & Missense & chr3:178936091 & $1633 \mathrm{G}>\mathrm{A}$ & E545K & intermediate & MSS \\
\hline \multirow[t]{2}{*}{10} & PIK3C2B & Missense & chr1:204400879 & $4198 A>C$ & T1400P & low & MSS \\
\hline & PIK3C2B & Missense & chr1:204399153 & $4294 \mathrm{~T}>\mathrm{C}$ & F1432L & low & MSS \\
\hline 11 & PIK3C2G & Missense & chr12:18793433 & $4130 \mathrm{G}>\mathrm{A}$ & R1377H & intermediate & MSS \\
\hline \multirow[t]{2}{*}{12} & PIK3C2B & Missense & chr1:204415223 & $2539 \mathrm{C}>\mathrm{A}$ & $\mathrm{H} 847 \mathrm{~N}$ & high & MSI-H \\
\hline & PIK3CA & Missense & chr3:178916905 & $292 \mathrm{~T}>\mathrm{C}$ & F98L & high & MSI-H \\
\hline 13 & PIK3C2B & Missense & chr1:204402506 & $3891 \mathrm{G}>\mathrm{T}$ & K1297N & intermediate & MSS \\
\hline 14 & PIK3C2B & Missense & chr1:204409404 & $3295 \mathrm{C}>\mathrm{T}$ & R1099C & intermediate & MSS \\
\hline 15 & PIK3C2G & Missense & chr12:18435626 & $611 \mathrm{C}>\mathrm{T}$ & S204F & intermediate & MSS \\
\hline 16 & PIK3C2B & CNA Amp & $\begin{array}{l}\text { chr1:204393979- } \\
204438930\end{array}$ & & & intermediate & MSS \\
\hline 17 & PIK3CA & CNA Amp & $\begin{array}{l}\text { chr3:178875270- } \\
178996445\end{array}$ & & & intermediate & MSS \\
\hline 18 & PIKЗCA & Missense & chr3:178921553 & $1035 T>A$ & N345K & low & MSS \\
\hline \multirow[t]{2}{*}{19} & PIK3C2B & Truncation & chr1:204438071 & 859_860insC & R287fs*38 & high & MSI-H \\
\hline & PIK3CA & Missense & chr3:178936091 & $1633 G>A$ & E545K & high & MSI-H \\
\hline 20 & PIK3C2G & Missense & chr12:18435612 & $597 \mathrm{C}>\mathrm{A}$ & N199K & low & MSS \\
\hline \multirow[t]{2}{*}{21} & PIK3C2G & Missense & chr12:18491478 & $1391 \mathrm{G}>\mathrm{C}$ & G464A & high & MSS \\
\hline & PIK3CA & Truncation & chr3:178951886 & $2941 C>T$ & Q981* & high & MSS \\
\hline 22 & PIK3C2B & Missense & chr1:204413250 & $2813 G>A$ & $\mathrm{R} 938 \mathrm{H}$ & intermediate & MSI-L \\
\hline 23 & PIK3C2B & Missense & chr1:204419142 & $2070 C>G$ & $1690 \mathrm{M}$ & low & MSS \\
\hline
\end{tabular}

weeks; HR=0.37. 95\%CI 0.13, 1.05). PDL1 expression had no significant effect on the radiologic response, but PFS improvement in patients with tumors expressing PDL1 trended towards statistical significance (median 18 vs. 40 weeks. $\mathrm{HR}=1.43$. 95\%CI 0.93, 4.46). BRAF mutation conferred shorter PFS (median 17 vs. 39 weeks. $\mathrm{HR}=0.35$. 95\% CI $0.14,0.91$ ) (figure 2).

Conclusions High tumor mutational burden and PIK3 mutation conferred better progression-free survival with immunotherapy across cancer types. The improvement in PFS in PIK3 mutated patients was independent of PDL1 status or TMB. The results should prompt further evaluation of these potential biomarkers and more widespread real-world data publications to help determine biomarkers that could benefit specific populations.

Ethics Approval The study was approved by University of Kentucky Institutional Review Board, approval number 49450 


\section{REFERENCES}

1. Topalian SL, Hodi FS, Brahmer JR, et al. Safety, activity, and immune correlates of anti-PD-1 antibody in cancer. N Engl J Med. 2012;366(26):2443-2454.

2. Spencer KR, Wang J, Silk AW, Ganesan S, Kaufman HL, Mehnert JM. Biomarkers for Immunotherapy: Current Developments and Challenges. Am Soc Clin Oncol Educ Book. 2016; 35:e493-503.

http://dx.doi.org/10.1136/jitc-2020-SITC2020.0053

\section{PATIENT STRATIFICATION USING CLINICAL PROTEOMICS - VALIDATED MULTIPLEXED MRM ASSAYS TO QUANTIFY HER2 AND OTHER BIOMARKERS IN CLINICAL FFPE TISSUES}

Maxim Isabelle*, Michael Schirm, Gwenaël Pottiez, Rudolf Guilbaud, Lorella Di Donato. Caprion Biosciences, Montreal, Canada

Background The advent of precision oncology has led a shift towards biomarker-driven clinical trial designs and molecular profiling of individual patients. Identification of patients with the target biomarker profile may be useful in guiding patient selection as an enrichment strategy for clinical trials. Targeted multiple reaction monitoring mass spectrometry (MRM-MS) analysis for multiplexed quantitation of biomarker proteins in FFPE tissue provides direct, more accurate and precise quantification over current 'gold standard' immunohistochemistry (IHC) methods. However, MRM-MS has not yet been broadly applied to clinical trials. In this study, we demonstrate the systematic development, optimization and analytical validation of quantitative, multiplexed MRM-MS assays for robust biomarker quantification in clinical FFPE tissues, including sample analysis under GCLP. Results from an MRM panel targeting 8 clinically relevant biomarker proteins will also be shown, including the measured HER2 levels in FFPE breast tumors classified by IHC as $0,1+, 2+$ or $3+$.

Methods MRM-MS biomarker panels were developed and optimized for multiplexed quantitation of $\leq 12$ proteins, in which unique peptides derived from each target protein were monitored as a surrogate measure of protein levels. Tumor regions from FFPE tissue sections were dissected using laser capture or macrodissection, solubilized, digested with trypsin to generate peptides for analysis, spiked with fixed levels of stable isotope labeled (SIL) peptide standards, and analyzed by MRM-MS. Analytical validation was performed per NCI CPTAC guidelines, including response curves, assay repeatability, selectivity, stability, and reproducibility of endogenous detection. Clinical performance was assessed using commercially sourced FFPE-tumor tissues, including a cohort of breast tumor tissues with a wide range of HER2 expression.

Results Assay performance results were protein/peptide dependent, with sensitivity in the low $\mathrm{pg} / \mu \mathrm{g}$ total protein range. For HER2, assay linearity was demonstrated over 2.5 to 3 orders of magnitude, with a precision and accuracy of $<15 \%$ over 3 independent runs. In sample analysis, the MRM-MS was sufficiently sensitive to detect HER2 in $1 \mu \mathrm{g}$ total protein from FFPE breast tumor classified by IHC as negative (0).

Conclusions GCLP-compliant quantitative multiplexed largescale clinical analysis of protein biomarkers by MRM-MS in FFPE tissue is feasible and enables precise and accurate quantitation of proteins when IHC methods are unsuitable or unavailable. Data can be used for patient stratification, optimization of treatment outcomes, drug resistance prediction, and to support clinical development of novel therapies.

http://dx.doi.org/10.1136/jitc-2020-SITC2020.0054

\section{THOUSANDS OF ANTIGENS ARE RECOGNIZED IN MICE VIA ENDOGENOUS ANTIBODIES AFTER BEING CURED OF A B78 MELANOMA VIA IMMUNOTHERAPY}

${ }^{1}$ Anna Hoefges*, ${ }^{1}$ Amy Erbe, ${ }^{1}$ Sean Mcilwain, ${ }^{1}$ Trang Le, ${ }^{1}$ Angie $\mathrm{Xu},{ }^{1}$ Nicholas Mathers,

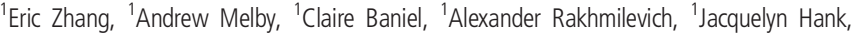
${ }^{2}$ Richard Pinapati, ${ }^{2}$ Brad Garcia, ${ }^{2}$ Jigar Patel, 'Zachary Morris, ${ }^{1}$ Irene Ong, ${ }^{1}$ Paul Sondel. ${ }^{1}$ University of Wisconsin Madison, Madison, WI, USA; ${ }^{2}$ Nimble Therapeutics, Madison, WI, USA

Background Antibodies can play an important role in innate and adaptive immune responses against cancer. Using a highdensity peptide array, we assessed potential protein-targets for antibodies detected in mice cured of melanoma through a combined immunotherapy regimen. Our goal was to determine the linear peptide sequences recognized by anti-tumor antibodies produced in mice cured of melanoma following immunotherapy.

Methods Mice with GD2-expressing syngeneic B78 melanoma were treated with a combination immunotherapy (local radiation therapy + intratumoral anti-GD2 mAb linked to IL2) capable of inducing an 'in situ vaccine' effect (ISV), enabling mice to be cured of their tumors with long-term immune memory. ${ }^{1}$ Naïve and immune sera were collected from these mice. Using flow cytometry, immune sera showed strong antibodybinding against B16 (parental cell line of B78 without GD2 expression). These sera were then used on a Nimble Therapeutics' peptide-array (either whole proteome or a curated list of $\sim 650$ proteins) to determine specific antibody-binding sites, and data were analyzed using a dynamic programming method that scans adjacent peptides to determine whether a peptide is bound by antibodies. Proteins were selected if peptides were bound using immune sera but not bound with the sera from naïve or non-responding tumor-bearing mice.

Results Multiple proteins were selectively identified by immune sera that were not detected by sera from naïve or nonresponding tumor-bearing mice. When focusing on the whole mouse proteome data, thousands of peptides were targeted by 2 or more mice and exhibited strong antibody binding only by immune sera. We also identified a few proteins that elicited an immune response in the naïve mouse sera that showed a significantly stronger signal in the immune sera of the same mice indicating that the cancer and/or the received therapy strengthened the immune response to these proteins.

Conclusions We are able to detect selective antibody binding to immune sera. However, we are continuing to refine our analytical methods and are further investigating the identified proteins. These peptides may potentially serve as targets for antibody-based or cellular therapies. In addition, we are examining whether some of the identified tumor-specific endogenous antibodies might be used as biomarkers to predict response to our ISV regimen and potentially other immunotherapy treatments.

\section{REFERENCE}

1. Morris ZS, et al. In Situ Tumor Vaccination by Combining Local Radiation and Tumor-Specific Antibody or Immunocytokine Treatments. Can Res. 2016; 76:3929-3941

http://dx.doi.org/10.1136/jitc-2020-SITC2020.0055 